\title{
Pharmacophore-Based Screening for the Successful Identification of Bio-Active Natural Products
}

\author{
Thierry Langer ${ }^{\star a, c}$, Christian Laggner ${ }^{\mathrm{a}}$, Judith M. Rollinger ${ }^{\mathrm{b}}$, and Hermann Stuppner ${ }^{\mathrm{b}, \mathrm{c}}$
}

\begin{abstract}
We report on the impact of pharmacophore-based virtual screening in the field of drug discovery from natural products. Confronted by an increasing number of secondary metabolites and an increasing number of biomolecular targets relevant in the therapy of human disorders, there is clearly the need for efficient data management. Filtering of compounds by virtual screening experiments already showed great effect when dealing with large libraries of potential bioactive molecules. Feature-based 3D pharmacophores have been successfully utilized for database mining in order to retrieve potentially bio-active molecules. However, for the discovery of natural lead candidates, the application of such in silico tools has been so far almost neglected. There seems to be several reasons for this. One concerns the scarce availability of natural product (NP) 3D databases in contrast to synthetic/combinatorial compound libraries; another reason might be the problematic compatibility of NPs with modern robotized high-throughput-screening (HTS) technologies. Additionally, the incalculable availability of pure natural compounds and their often too complex chemistry is claimed to hamper such an approach. Thus, research in this field appears time-consuming, highly complex, expensive, and ineffective. Nevertheless, naturally derived compounds are still among the most favorable sources of new drug candidates. A more rational and economic search for new lead structures originating from nature must therefore be a priority in order to overcome these problems.
\end{abstract}

Keywords: Natural products · Pharmacophores · Virtual screening

${ }^{*}$ Correspondence: Dr. T. Langera,c

Tel.: +435125075252

Fax: +435125075269

E-Mail: Thierry.Langer@uibk.ac.at

anstitute of Pharmacy / Pharmaceutical Chemistry

Leopold-Franzens University of Innsbruck

Innrain 52c

6020 Innsbruck

Austria

bInstitute of Pharmacy / Pharmacognosy

Leopold-Franzens University of Innsbruck

Inte:Ligand $\mathrm{GmbH}$, Software Engineering and Con-

sulting

Clemens Maria Hofbauergasse 6

2344 Maria Enzersdorf

Austria

\section{Introduction}

In modern drug discovery we are confronted by a paradoxical situation: Advanced technological and molecular know-how, e.g. in the area of genomics, combinatorial chemistry, high-throughput-screening (HTS), robotized and miniaturized process cycles were introduced as highly efficient tools in pharmaceutical industry. These costly procedures were expected to raise the number of launched drug substances; however the results have been disappointing and pharma still faces major challenges. ${ }^{[1]}$ Despite increasing research and development expenses of the leading pharma companies the number of new drugs on the market has fallen steadily in recent years. ${ }^{[2]}$

On the other side we are faced by a high traditional impact of naturally derived medicines and incredible success stories of natural products (NPs) as potent remedies from the beginnings of human therapeutic activity to modern research and drug development Nevertheless, most of the large pharmaceutical companies scaled down or terminated their work in NPs operations. The reason behind this phenomenon is principally that the drug discovery process starting from natural sources is hardly compatible with the today's highly automatized drug discovery technologies. Thus, the pre-eminence of combinatorial chemistry as the preferred method for generating new drug leads has led to the comparative neglect of this valuable resource. In a recent updated review ${ }^{[3]}$ of an older study, ${ }^{[4]}$ Newman and Cragg summarize the role of natural products as sources of new drugs over the last 25 years concluding that, from 1010 new chemical entities (NCE) investigated, $43(4.25 \%)$ stem directly from natural products, $232(22.97 \%)$ are direct derivatives from NPs, and another $108(10.69 \%)$ bear the pharmacophore of a natural compound. The fact that in total almost $38 \%$ of NCE have some origins in NPs unequivocally underlies the importance of such compounds in modern drug discovery and development. Strohl summarized the difficulties of NP programs versus synthetic chemicals. ${ }^{[5]}$ These include

i) the existence of known potent antimicrobial and antiproliferative NPs and the lack of sufficient dereplication programs which prevent their repeated discovery;

ii) the fact that - in contrast to the highly sophisticated molecular targets - NP 
extracts are generally regarded as too 'dirty', too difficult to assay, and too time-consuming to work with;

iii) obtaining an assay hit resulting from a bio-guided fractionation, the structure of the NP still has to be elucidated compared with synthetic chemicals;

iv) NPs are often deemed as too structurally complex.

Strohl nevertheless concludes that applying an active NP program is an "expensive endeavour" which, however, is "well worth the cost".

\section{Status of NPs}

To date some 200,000 natural compounds ${ }^{[6-8]}$ are published. The terrestrial flora has been intensively investigated during the last decades, the potential in finding new NPs slumbering in untapped biota is however nearly inconceivable. It is estimated that only 5 to $15 \%$ of the approximately 250,000 high plant species described have ever been in the focus of phytochemical and pharmacological investigations. [9] This percentage is even lower in the field of fungal (less than 5\%) and bacterial species (less than 1\%). ${ }^{[10]}$ The most interesting fact in this respect is that NPs contain an inherently large-scale of structural diversity. Thus, about $40 \%$ of the chemical scaffolds of published NPs are unique and have not been made by any chemist.[11]

\section{Computational Approaches for the Discovery of Lead Structures from Nature}

The increased understanding of fundamental principles of protein-ligand interactions and the steadily growing number of 3D-structures of potential and experimentally proven ligands provide excellent possibilities toward more rationalized concepts in drug discovery. In the area of medicinal chemistry, computational methods, like virtual screening experiments, have already proved to be successful approaches. They are needed to exploit the available structural information, to understand specific molecular recognition events, and to clarify the function of the target macromolecule. Although rationalized procedures in the search for bioactive natural products are in great demand to find the 'needles in the haystack', computational assistance has barely been used in natural product research.

The common idea of all computational approaches within the early drug discovery process is to mine more or less large compound databases in silico and to select a limited number of candidates proposed to have the desired biological activity. For this process the term 'data mining' was coined. ${ }^{[12,13]}$ The key goal of the use of such methods is to reduce the overall cost associated with the discovery and development of a new drug, by identifying the most promising candidates to focus experimental efforts on. Recently published reviews and books on the impact of computational chemistry for lead structure determination highlight such efforts. ${ }^{[14-20]}$

If the 3D structure of the biological target is known, high-throughput docking turns out to be a valuable structure-based virtual screening method. ${ }^{[21-23]}$ Within this context, the scoring of hits retrieved still remains a question that is often discussed. In fact, the major weakness of docking programs currently lies not in the docking algorithms themselves but still in the inaccuracy of the functions that are used to estimate the affinity between ligand and target, the so-called scoring functions. ${ }^{[24,25]}$

Since there is still a lack of comprehensive NP 3D libraries, the number of virtual screening studies published for the rational access to bioactive NPs is limited. Some examples using docking as a structure-based virtual screening tool include papers from Liu and Zhou, ${ }^{[26]}$ Toney et al., ${ }^{[27]}$ Cozza et al., ${ }^{[28]}$ Zhao and Brinton, ${ }^{[29]}$ and Liu et al. ${ }^{[30]}$ Compound databases containing ingredients used in traditional Chinese medicine (TCM) have been used in several cases in such applications.[30,31]

\section{The Pharmacophore Concept in NP Research}

The pharmacophore concept has proven to be extremely successful, not only in rationalizing structure-activity relationships, but also by its large impact in developing the appropriate 3D-tools for efficient virtual screening. ${ }^{[32]}$ The profiling of combinatorial libraries and the classification of compound series are other often-used applications of this concept. Although well established in combinatorial chemistry, it has to be pointed out that the tools described in this section have likewise a considerable impact on the rational finding of new potential lead compounds originating from the immense source of secondary metabolites. The prior use of pharmacophore models in biological screening of NPs is an efficient procedure since it eliminates quickly molecules that do not possess the required features, thus leading to a dramatic increase of enrichment, when compared to a purely random screening experiment. Doman et al. found only 85 molecules or $0.021 \%$ active as protein tyrosine phosphatase-1B inhibitors $\left(\mathrm{IC}_{50}<100 \mu \mathrm{M}\right)$ by a HTS of approximately 400,000 compounds. ${ }^{[33]}$ On the other hand, of 365 molecules suggested by molecular docking, 127 or $34.8 \%$ were found to be active. Thus, docking-based virtual screening enriched the hit rate by almost 1700 -fold over random screening.

It has to be pointed out, however, that additional molecular characteristics not reflected by pharmacophore models (physico-chemical properties relevant for ADME and toxicological properties) must be taken into account when deciding upon which compounds should be further developed. [34] A rapid identification and elimination of compounds with unsuitable physicochemical and pharmacokinetic properties is a pivotal step in the early drug discovery process. ${ }^{[35,36]}$ This must be considered for synthetics as well as NPs, though studies revealed secondary metabolites not only high scaffold diversity; biosynthesized molecules also show structural and spatial characteristics that are closer to drug leads than those of synthetic molecules. ${ }^{[37,38]}$ Typically, NPs include more chiral centers and their stereochemical architecture is much more complex than that of synthetic molecules. Furthermore, they usually contain more carbon, hydrogen and oxygen atoms, however, fewer nitrogen and other atoms were present compared to synthetics. NPs often show a molecular weight higher than 500 Da combined with a high polarity which is in clear contrast to Lipinski's 'Rule of Five'. ${ }^{[39]}$ Nevertheless only about $10 \%$ of NPs contain two or more violations of Lipinski's rules. ${ }^{[38]}$ Thus natural products can be seen as containing highly diverse scaffolds endowed with potential drugable pharmacophores.

\section{Structure-based Pharmacophore Modeling}

An inevitable prerequisite for generating a structure-based model is knowledge about the ligand-target interaction ${ }^{[40]}$ including the availability of the 3D structure of the target either by X-ray crystallography or NMR or even constructed on the basis of homologous proteins. A new software tool (LigandScout) has recently been described for the successful automated generation of chemical feature-based pharmacophore models starting from ligand-target 3D structures. ${ }^{[41]}$ The performance of this program allows the detection of relevant interaction points between ligand and protein. The binding mode of the ligand in the active site of a protein can be visualized in a sophisticated way. In a study published previously, this software was used for the detection and interpretation of crucial interaction patterns between ligands and the factor Xa protein structure[42] (Fig. 1). In a second step, the program Catalyst, ${ }^{[43]}$ a state of the art virtual screening platform, was used for rapid virtual screening of multiconformational 3D structure databases in order to retrieve selectively a $78 \%$ fraction 
of the known factor Xa inhibitors within a small subset of the large Derwent World Drug Index library. ${ }^{[44]} \mathrm{A}$ further application of the LigandScout pharmacophore definitions covers the rationalized search for angiotensin converting enzyme (ACE)-2 inhibitors by virtual screening of approximately 3.8 million compounds from various commercial databases. ${ }^{[45]}$ Hit reduction and selection was achieved using a five feature hypothesis based on a recently resolved inhibitor-bound ACE2 crystal structure. Seventeen virtual hits were selected for their experimental validation in a bioassay; the concept was confirmed since all of them were revealed as ACE-2 inhibitors, all representing new chemotypes.

Barreca and co-authors developed a 3D structure-based pharmacophore model with LigandScout for the discovery of new scaffolds acting as HIV-1 non-nucleoside reverse transcriptase inhibitors by virtual screening of large chemical databases. Six virtual hits were finally selected for determination of their inhibitory effects. Those belonging to the new scaffold class of the quinolin-2(1H)-one family exhibited reverse transcriptase inhibitory activity at sub-micromolar concentrations. ${ }^{[46]}$

In a recently published work, Schuster et al. described a so-called cytochrome P450 profiler. ${ }^{[4]}$ Several structure-based and ligand-based pharmacophore models for substrates and inhibitors of five cytochrome P450 isoenzymes (1A2, P450 2C9, $\mathrm{P} 450$ 2C19, P450 2D6, and P450 3A4) were created and validated by the authors. Their results showed that the models were suitable for fast pharmacokinetic profiling of large drug-like databases.

In this context the parallel screening approach is of particular interest. Whereas in usual virtual screening cycles interactions of thousands or even millions of 3D database entries are browsed against one pharmacophore model, in the case of parallel screening, low-energetic conformers of one structure are screened for their potential interactions against numerous models. The basics of parallel screening have just recently been presented by Steindl and coauthors. ${ }^{[48-50]}$ This in silico concept is of particular interest to virtually scrutinize drug candidates for their preliminary activity profiling relevant to putative side effects and toxicity. ${ }^{[32]}$ According to the obtained interactions to virtually screened antitargets (e.g. hERG, alpha, D2, D3, H1, I2, A2A, A2B, cytochrome P 450, etc.) a first insight to potentially risky affinities is provided before time- and cost-intensive toxicological studies are performed.

The virtual screening approach using a structure-based pharmacophore model has revealed some first application examples in NP research: Nikolovska-Coleska and coauthors successfully pursued this in silico

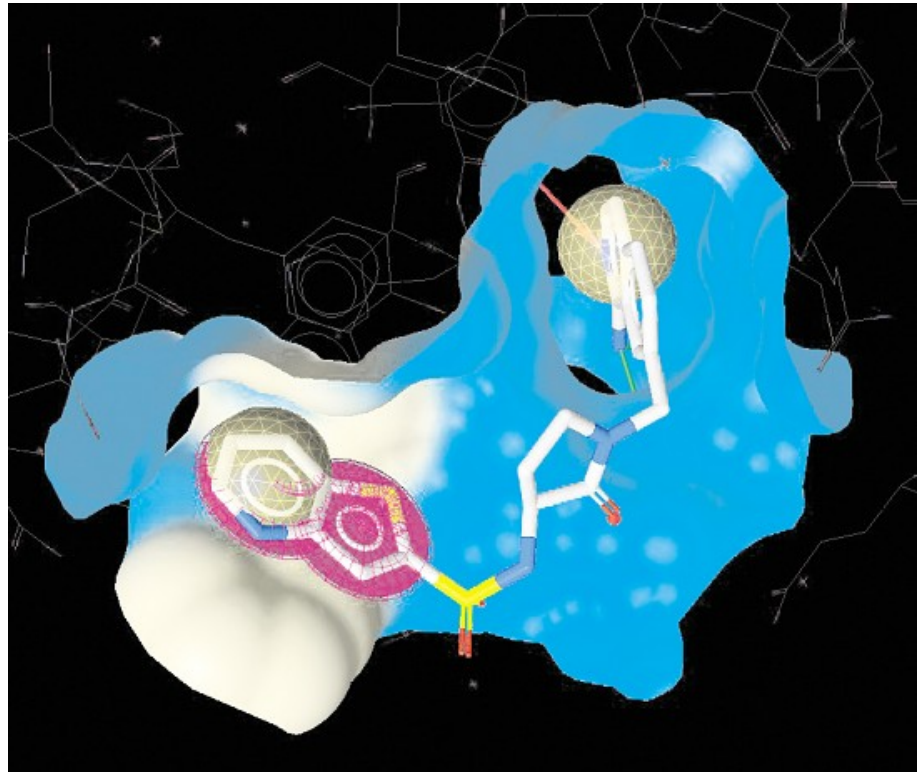

Fig. 1. Chemical feature-based pharmacophore representation of a ligand bound to its active site using the LigandScout visualization and pharmacophore modeling platform (Ligand: RPR208815, Protein: Factor Xa, PDB entry 1for) strategy in the area of X-linked inhibitors of apoptosis (XIAP). ${ }^{[51]}$ A high-resolution 3D structure of the XIAP BIR3 domain complexed with the N-terminal end of the Smac/Diablo protein, ${ }^{52]}$ which is an endogenous ligand of the respective XIAP binding pocket, was used as the starting point to virtually screen an in-house 3DNP database. In our group, we previously focused on acetylcholinesterase (AChE); ;53] according to the cholinergic hypothesis of the pathogenesis of Alzheimer's disease, inhibitors of the AChE are successfully used as therapeutic strategy. Based on the co-crystal structure of AChE with its ligand galanthamine, a structure-based pharmacophore model was generated and used for an in silico screening of a multi-conformational database consisting of more than 110,000 NPs. From the obtained hit list, promising, virtually active candidates shown in Fig. 2 were selected, namely scopoletin (1) and its

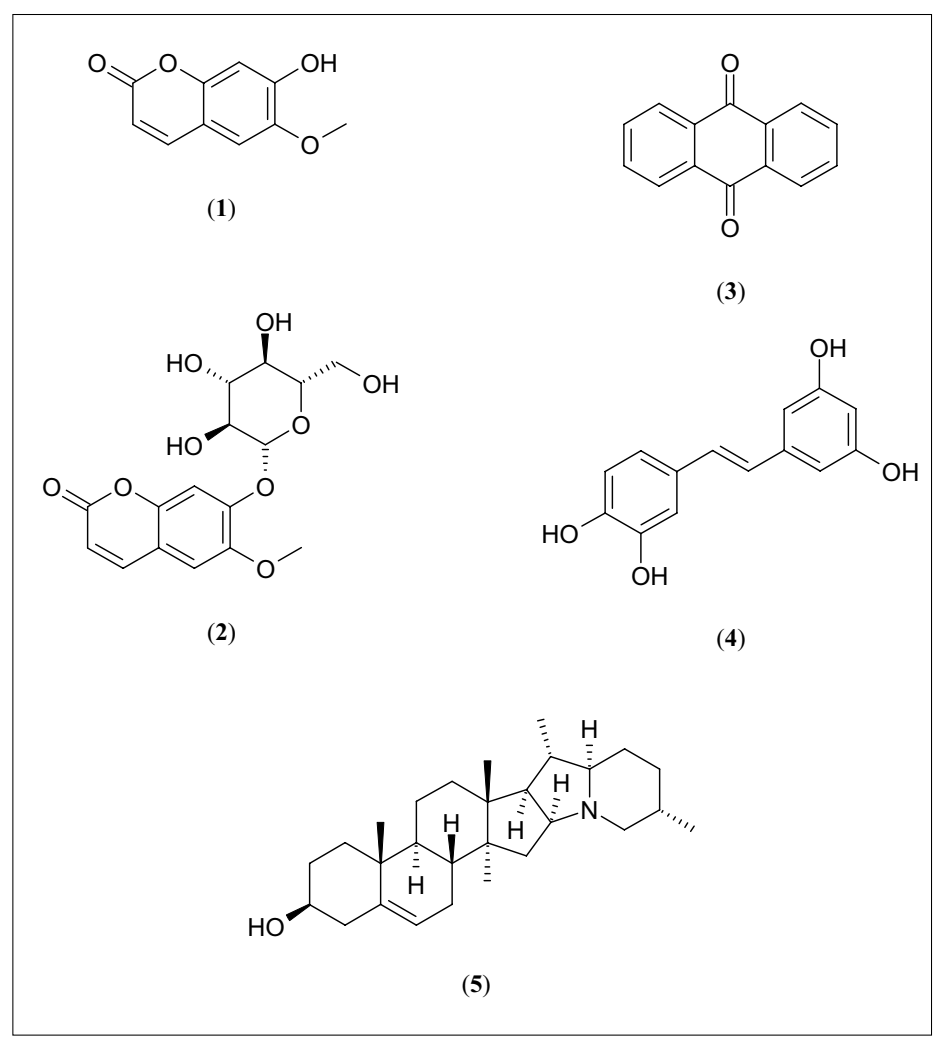

Fig. 2. 
glucoside scopolin (2). Their AChE inhibitory effect was first verified from the crude extract of Scopolia carniolica roots using a bioautographic TLC-assay. The isolated coumarins showed a significant and dosedependent inhibition of the AChE in the microplate enzyme assay as well as in the in vivo test. The i.c.v. application of both coumarins on rats resulted in a long-lasting, pronounced and - in the case of the glucoside - even in a 2-fold higher increase of the neurotransmitter's concentration than the one caused by the positive control galanthamine.

\section{Ligand-based Pharmacophore Modeling}

Very often, lead discovery projects have reached already a well-advanced stage before detailed structural data on the protein target has become available, even though it is well recognized that modern methods of molecular biology together with biophysics and computational approaches enhance the likelihood of successfully obtaining detailed atomic structure information. A possible consequence is that often scientists identify and develop novel compounds for a target using preliminary structure-activity information, together with theoretical models of interaction. Only responses that are consistent with the working hypotheses contribute to an evolution of the used models. Within this framework, the chemical feature-based pharmacophore approach has also proven to be successful ${ }^{[54]}$ allowing the perception and understanding of key interactions between a receptor and a ligand on a generalized level, starting from extensive conformational analysis of active ligand molecules and performing superpositioning experiments in order to find the common elements responsible for binding and triggering a biological response (Fig. 3). Such ligand-based pharmacophore models together with large 3D structure databases originating either from in house compound collections, from commercial vendors, or from natural products databases have proven to be as useful as structure-based models for in silico screening experiments. When using ligand-based pharmacophore models as screening filters instead of protein 3D structures, affinity estimation is only based on geometric fit of compound atoms or groups to features of the model. In these cases, the values calculated are often far away from reality, however, still are useful for filtering possible hits from non-binding molecules. Additionally, in pharmacophore fitting procedures, calculation demands are considerably lower than in docking algorithms allowing the number of compounds to be processed in the same time to be by far higher than even in high-throughput dock-

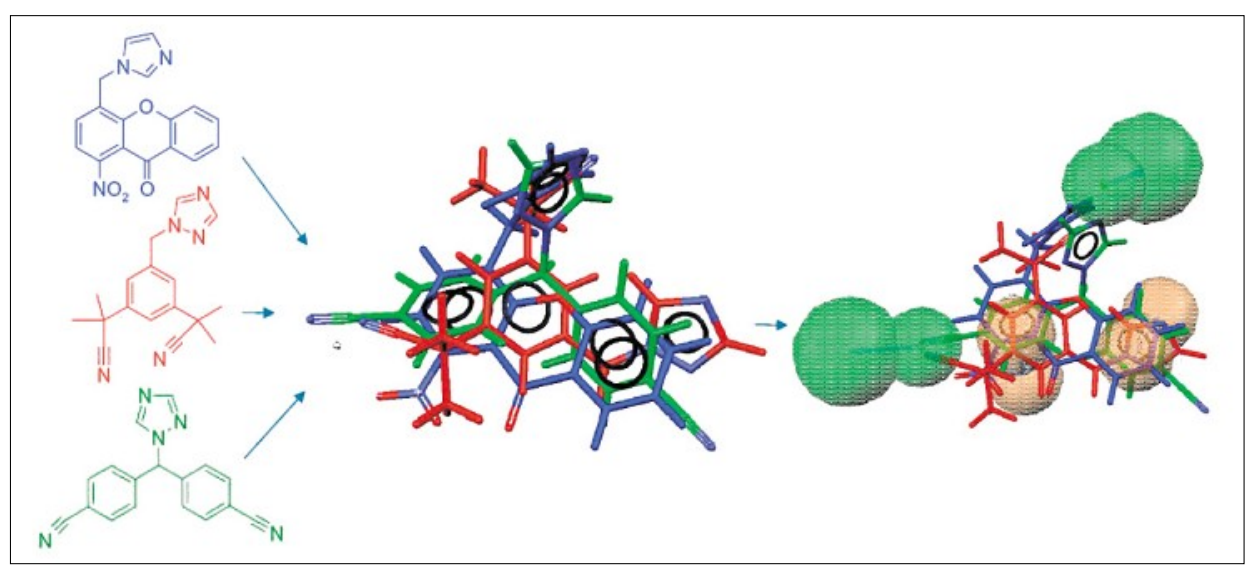

Fig. 3: Concept for generating a ligand-based pharmacophore model using the Catalyst program: a) selection of bio-active ligands; b) alignment of low-energetic conformers of the selected ligands; c) derivation and determination of common pharmacophore features

ing. Several reviews covering successful applications of such feature-based methods have been published by Kurogi et al., ${ }^{[55]}$ by Langer and Krovat, ${ }^{[54]}$ and by Güner et al. ${ }^{[56]}$ They outline the theoretical background and describe several significant studies including 3D database search strategies.

In the field of NPs only a very limited number of studies report from the rationalized access to bioactive compounds via ligand-based virtual screening. For example, this method was pursued for the discovery of inhibitors of the COP9 signalosome (CNS) associated kinases CK2 and PKD. ${ }^{[57]}$ Using NPs curcumin and emodin as lead structures, a virtual screening of an in-house database was carried out. Among the virtual hits seven NPs, e.g. anthraquinone (3) and piceatannol (4), were found to significantly induce apoptosis by inhibition of the CSN-associated kinases using in vitro and cell culture experiments. A further study has demonstrated the power of the ligand-based approach applied to pharmacophore modeling of sigma-1 ligands. ${ }^{[58}$ Therein, some reliable pharmacophore models could be extracted solely from ligand structure information. Compounds with potent affinities to the sigma- 1 receptor known from literature were structurally aligned to derive distinct common features. Their 3D arrangement in combination with a spatial restriction was then used for the generation of a pharmacophore model, which was able to retrieve compounds with high affinity values, among them also NPs, like solanidine (5).

\section{Conclusion}

When analyzing our drug discovery adventure starting from natural products we are facing two facts:

i) statistics show that the myriad of structurally diverse natural compounds are the most favored source of new drugs for clinical use; $[3,4]$

ii) the drug discovery process has moved toward more rational concepts based on the increasing understanding of the molecular principles of protein-ligand interactions.

Driven by economic interest, fundamental advances have been made in research applying data mining strategies, like pharmacophore-based in silico screening.

Though being aware of both potentials, their combined benefit so far could only be savored to a rudimentary extent. Only limited attempts applying innovative in silico tools in NP research are pursued, mainly because the search for bioactive compounds is a complex and multi-disciplinary challenge. Thus, a sensible adaptation of computational strategies is in demand to profit in an economic way from the unique chemical and biological diversity associated with NPs. Examples underlining the impact of virtual screening on the identification of active NPs have been presented in this survey. Though the full potential in this field is by far not tapped, these early results indicate that the integrated virtual screening approaches are target-oriented and trendsetting strategies. However, as for any computer-based technique, the successful use of virtual screening will entirely depend on the way it is utilized and the quality of its underlying experimental data. It has to be stressed that virtual screening techniques must not be used exclusively as activity-predicting tools, since the results provide merely an indication for a putative activity: it is only by the creation of interfaces between computational tools and well-established methods from pharmacognosy that a reasonable standard of success can be achieved. ${ }^{[59-61]}$ The search for the most effective strategy is best performed by a drug discovery process that involves the exploitation of all the information which can be gathered from 
bioactivity-guided fractionation, on-line analytical activity profiling, ethnopharmacological screening, chemoinformatics, virtual and in vitro screening studies. In the first instance it behoves the represents of modern pharmacognosy to skillfully exploit knowledge from all these fields because it is of paramount importance to sift through the enormous wealth of NPs.

Received: April 10, 2007

[1] S. Frantz, Nat. Rev. Drug Discov. 2007, 6, 5.

[2] A. Smith, Nature 2002, 418, 453.

[3] D. J. Newman, G. M. Cragg, J. Nat. Prod. 2007, 70, 461 .

[4] D. J. Newman, G. M. Cragg, K. M. Snader, J. Nat. Prod. 2003, 66, 1022.

[5] W. R. Strohl, Drug Disc. Today 2000, 5, 39.

[6] 'Dictionary of Natural Products' provided by Chapman \& Hall, CRC, http://www. crcpress.com/, accessed in April 2007.

[7] 'Strasburger - Lehrbuch der Botanik', Eds. P. Sitte, E. W. Weiler, J. W. Kadereit, A. Bresinsky, C. Körner, 35. Auflage, Spektrum Akademischer Verlag, Heidelberg, Berlin, 2002, 339.

[8] M. Tulp, L. Bohlin, Bioorg. Med. Chem. 2005, 13, 5274.

[9] M. Balandrin, Am. Chem. Soc. Symposium Series 1993, 534, 2.

[10] G. M. Cragg, D. J. Newman, K. M. Snader, J. Nat. Prod. 1997, 60, 52.

[11] T. Henkel, R. M. Brunne, H. Muller, F. Reichel, Angew. Chem., Int. Ed. 1999, 38, 643.

[12] J. Morrell, Book Abstr 211th ACS National Meeting 1996, CINF-052.

[13] J. Gasteiger, A. Teckentrup, L. Terfloth, S. Spycher, J. Phys. Org. Chem. 2003, 16, 232.

[14] J. Xu, A. Hagler, Molecules 2002, 7, 566.

[15] 'Virtual Screening in Drug Discovery', Eds. A. Alvarez, B. Shoichet, Taylor \& Francis, CRC-Press, Boca Raton, 2005.

[16] T. Langer, R. D. Hoffmann, Curr. Pharm. Des. 2001, 7, 509.

[17] 'Pharmacophores and Pharmacophore Searches', Eds. T. Langer, R. D. Hoffmann, in 'Methods and Principles in Medicinal Chemistry', Vol. 32, Wiley-VCH, Weinheim, 2006.

[18] 'Virtual screening for bioactive molecule', Eds. H. J. Böhm, G. Schneider, H. Kubinyi, R. Mannhold, H. Timmerman, in 'Methods and Principles in Medicinal Chemistry', Wiley, New York, 2000.

[19] C. J. Manly, S. Louise-May, J. D. Hammer, Drug Discov. Today 2001, 6, 1101.

[20] E. M. Krovat, T. M. Steindl, T. Langer Curr. Comput.-Aided Drug Des. 2005, 1, 93.

[21] R. Abagyan, M. Totrov, Curr. Opin. Chem. Biol. 2001, 5, 375.

[22] G. Schneider, H. J. Böhm, Drug Disc. Today 2002, 7, 64
[23] B. K. Shoichet, S. L. McGovern, B. Wei, J. J. Irwin, Curr. Opin. Chem. Biol. 2002, $6,439$.

[24] M. Stahl, M. Rarey, J. Med. Chem. 2001, 44, 1035.

[25] G. L. Warren, C. W. Andrews, A.-M. Capelli, B. Clarke, J. LaLonde, M. H. Lambert, M. Lindvall, N. Nevins, S. F. Semus, S. Senger, G. Tedesco, I. D Wall, J. M. Woolven, C. E.; Peishoff, M. S. Head, J. Med. Chem. 2006, 49, 5912.

[26] B. Liu, J. Zhou J. Comp. Chem. 2005, 26 , 484.

[27] J. H. Toney, S. Navas-Martin, S. R. Weiss, A. Koeller, J. Med. Chem. 2004, 47, 1079 .

[28] G. Cozza, P. Bonvini, E. Zorzi, G. Poletto, M. A. Pagano, S. Sarno, A. Donella-Deana, G. Zagotto, A. Rosolen, L. A. Pinna, F. Meggio, S. Moro, J. Med. Chem. 2006, 49, 2363.

[29] L. Zhao, R. D. Brinton, J. Med. Chem. 2005, 48, 3463

[30] H. Liu, Y. Li, M. Song, X. Tan, F. Cheng, S. Zheng, J. Shen, X. Luo, R. Ji, J. Yue, G. Hu, H. Jiang, K. Chen, Chem. Biol. 2003 , 10,1103

[31] T. M. Ehrmann, D. J. Barlow, P. J. Hylands, J. Chem. Inf. Model. 2007, 47, 254.

[32] T. Langer, R. D. Hoffmann, Exp. Opin. Drug Discov. 2006, 1, 261.

[33] T. N. Doman, S. L. McGovern, B. J. Witherbee, T. P. Kasten, R. Kurumbail W. C. Stallings, D. T. Connolly, B. K. Shoichet, J. Med. Chem. 2002, 45, 2213.

[34] D. S. Samiulla, V. V. Vaidyanathan, P. C. Arun, G. Balan, M. Blaze, S. Bondre, G. Chandrasekhar, A. Gadakh, R. Kumar, G. Kharvi, Mol. Divers. 2005, 9, 131.

[35] B. Testa, Adv. Drug Res. 1984, 13, 1.

[36] 'Pharmacokinetics and Metabolism in Drug Design', Eds. D. A. Smith, H. van de Waterbeemd, D. K. Walker, in 'Methods and Principles in Medicinal Chemistry', 2nd ed, Wiley-VCH, Weinheim, 2006

[37] M. Feher, J. M. Schmidt, J. Chem. Inf. Comput. Sci. 2003, 43, 218.

[38] F. Stahura, J. W. Godden, X. Ling, J. Bajorath, J. Chem. Inf. Comput. Sci. 2002, 40 1254.

[39] C. A. Lipinski, F. Lombardo, B. W. Dominy, P. J. Feeney, Adv. Drug Deliv. Rev. 1997, 23, 3 .

[40] M. Congreve, C. W. Murray, T. L. Blundell, Drug Disc. Today 2005, 10, 895.

[41] G. Wolber, T. Langer, J. Chem. Inf. Model. 2005, 45, 160. LigandScout 1.03 is available from Inte:Ligand $\mathrm{GmbH}$, Austria, www.inteligand.com

[42] E. M. Krovat, K. H. Frühwirth, T. Langer J. Chem. Inf. Model. 2005, 45, 146.

[43] Catalyst 4.11, available from Accelrys Inc, San Diego, CA, USA, www.accelrys.com.

[44] 'The Derwent Word Drug Index' by Thomson Scientific, available in Catalyst 3D data format from Accelrys Inc., San Diego, CA, USA, www.accelrys.com.
[45] M. Rella, C. Rushworth, J. L. Guy, A. J. Turner, T. Langer, R. M. Jackson, J. Chem. Inf. Model. 2006, 46, 708 .

[46] M. L. Barreca, L. De Luca, N. Iraci, A. Rao, S. Ferro, G. Maga, A. Chimirri, $J$. Chem. Inf. Model. 2007, 47, 557.

[47] D. Schuster, C. Laggner, T. M. Steindl, T Langer, Curr. Drug Discov. Technol. 2006 3,1 .

[48] T. M. Steindl, D. Schuster, C. Laggner T. Langer, J. Chem. Inf. Model. 2006, 46 , 2146.

[49] T. M. Steindl, D. Schuster, G. Wolber, C. Laggner, T. Langer, J. Comput.-aided Mol. Des. 2006, 20, 703.

[50] T. M. Steindl, D. Schuster, C. Laggner, K. Chuang, R. D. Hoffmann, T. Langer, $J$. Chem. Inf. Model. 2007, 47, 563.

[51] Z. Nikolovska-Coleska, L. Xu, Z. Hu, Y. Tomita, P. Li, P. P. Roller, R. Wang, X. Fang, R. Guo, M. Zhang, J. Med. Chem. 2004, 47, 2430.

[52] G. Wu, J. Chai, T. L. Suber, J. W. Wu, C. Du, X. Wang, Y. Shi, Nature 2000, 408, 1008

[53] J. M. Rollinger, A. Hornick, T. Langer, H. Stuppner, H. Prast, J. Med. Chem. 2004, 47, 6248.

[54] T. Langer, E. M. Krovat, Curr. Opin. Drug Discov. Dev. 2003, 6, 370.

[55] Y. Kurogi, O. F. Güner, Curr. Med. Chem. 2001, 8, 1035.

[56] O. Güner, O. Clement, Y. Kurogi, Curr. Med. Chem. 2004, 11, 763 .

[57] M. Füllbeck, X. Huang, R. Dumdey, C. Frommel, W. Dubiel, R. Preissner, BMC cancer 2005, 5, 97.

[58] C. Laggner, C. Schieferer, B. Fiechtner, G. Poles, R. D. Hoffmann, H. Glossmann, T. Langer, F. Moebius, J. Med. Chem. 2005 , $48,4754$.

[59] J. M. Rollinger, T. Langer, H. Stuppner, Curr. Med. Chem. 2006, 13, 1491.

[60] J. M. Rollinger, T. Langer, H. Stuppner, Planta Med. 2006, 72, 671

[61] J. M. Rollinger, A. Bodensieck, C. Seger, E. P. Ellmerer, R. Bauer, T. Langer, H. Stuppner, Planta Med. 2005, 71, 399. 